\title{
A Preliminary Study in Neonatal Cardiorespiratory Monitoring through Diaphragmatic Electromyography
}

\author{
Marcela Tobón-Cardona ${ }^{1}$, Tapio Seppänen ${ }^{2}$ and Róbinson Torres ${ }^{1}$ \\ ${ }^{1}$ Biomedical Engineering Research Group EIA - CES (GIBEC), EIA University, Envigado, Colombia \\ ${ }^{2}$ Center for Machine Vision and Signal Analysis, University of Oulu, Oulu, Finland
}

\begin{abstract}
Cardiorespiratory monitoring continues to be a challenge in the Neonatal Intensive Care Unit (NICU) due to the sensitivity of current techniques to false alarms and artifacts. Surface diaphragmatic electromyography (EMGdi) can be used to measure directly the respiratory muscles activity and to reduce motion artifacts. We propose to acquire ECG and EMGdi with the electrodes placed on the diaphragm to measure heart and respiratory rate in neonates. Data from fourteen neonates was analyzed to compare the measurements of the signal acquired in the diaphragm with the ECG bipolar leads and a reference respiration signal from piezoelectric respiratory effort belt. Bland-Altman plots showed agreement between heart rate monitoring in the diaphragm with no significant differences. Detection of breaths showed similar results in the EMGdi derived wave compared with the reference signal. Thus, heart and respiration rate monitoring in neonates can be accomplished with ECG and EMG signals acquired from the diaphragm.
\end{abstract}

Keywords - Monitoring, Electrocardiography, Respiration, Electromyography, Diaphragm.

\section{INTRODUCTION}

Cardiorespiratory monitoring is performed every day in the Neonatal Intensive Care Unit (NICU). The techniques most commonly used in medical devices are the electrocardiogram (ECG) and the respiratory impedance pneumography (IPG), which utilizes ECG electrodes [1]. However, IPG is sensitive to movement by the patient and obstruction of the respiratory tract can give erroneous results [2]. Thus, several techniques have been proposed to monitor the breathing signal based on movement, volume and tissue composition detection; airflow sensing, blood gas measurement [3] and non-contact respiration monitoring [4]. Nevertheless, convincing evidence of the clinical usefulness of respiratory monitors is still lacking [3] and respiratory rate monitoring remains largely dependent on manual observations [2]. As a result monitoring of heart rate and respiration continues to be a challenge in the NICU setting with the need of methods to monitor cardiorespiratory activity that is resistant to false alarms and can be implemented continuously and noninvasively [5].
Acquiring respiration signal using surface diaphragmatic electromyography (EMGdi) has been proposed to measure directly the respiratory muscles activity with the advantage to reduce motion artifacts, estimate airway obstruction [6] and detect apnea in neonates [7]. However, cardiorespiratory monitoring acquiring ECG and EMGdi would require surface electrodes placed on the diaphragm of the patient combined with the ECG electrodes, which is not feasible in neonates due to the size of the newborn, especially if they are premature. Therefore, we propose to acquire both signals with the electrodes placed on the diaphragm to monitor heart and respiration rate in neonates.

\section{MATERIALS AND METHODS}

\section{A. Data Collection}

ECG and EMGdi signals were collected in the Neonatal Intensive Care Unit of Hospital General de Medellín Luz Castro de Gutiérrez, Colombia with the approval of the ethics committee of CES University (Medellín, Colombia) and following the Helsinki Declaration of 1975. Thirty subjects, between one and fifty-six days of born, participated in the study with the informed consent of the patient's legal representative. Fourteen of them are considered in this paper. Improper electrode location, data suspended by the parents, the presence of non-normal breathing patterns and other artifacts were the reasons to not include all the subjects in the present study. The cause of electrodes mislocation was the presence of the vital sign sensors connected to the newborn, which cannot be disconnected from the neonate in the NICU, and were interfering with the electrodes location for the study. Non-normal breathing patterns like respiratory pauses were neither included in the analysis.

Signals were collected with ECG surface electrodes placed on the diaphragm of the newborn where imaginary vertical lines with the nipples cross the costal margin in the right (R) and left side (L) of the torso [6], and a reference electrode (GND) located in the sternum (Fig. 1). This electrode location was used to obtain the EMGdi signal and the ECG from the diaphragm. For the purposes of comparison, 
additional electrodes were placed on the limbs of the neonate to acquire the standard bipolar ECG leads (Lead I, Lead II and Lead III); and a belt piezoelectric sensor (g.RESPsensor from g.tec, Schiedlberg, Austria) was connected on the abdomen, to acquire the reference respiration signal of the newborn. Data acquisition was accomplished using a biosignal amplifier (g.USBamp from g.tec, Schiedlberg, Austria) for acquisition of electrophysiological signals with a sampling rate of $1200 \mathrm{~Hz}$ and allowing the simultaneous acquisition of the bipolar leads, the ECG in the diaphragm, the EMGdi wave and the belt respiration signals.

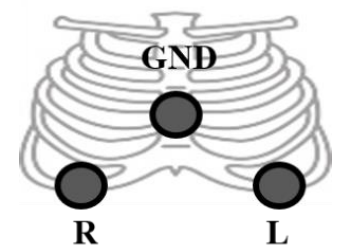

Figure 1. Electrodes position to acquire ECG and EMGdi signals for cardiorespiratory monitoring in neonates.

\section{B. Signal Processing}

Processing of the data was done in Matlab. Fourth order Butterworth filters were implemented in the ECG signals with $3 \mathrm{~dB}$ cutoff frequencies $0.5 \mathrm{~Hz}$ and $40 \mathrm{~Hz}$. 100 seconds of data in each subject were analyzed to obtain the RR intervals in each lead and R-peak detection was accomplished using an adaptation from the Pan-Tompkins detector [8], [9]. Once the QRS complexes were detected, visual validation was done to identify and correct the misdetected beats.

The respiration signal derived from EMGdi was obtained with digital processing. The major problem with EMGdi signals recorded by surface electrodes is ECG interference [10]. Different techniques had been proposed to reduce the cardiogenic interference in EMG [10]. Nevertheless, the interest in this case with the EMGdi signal was to obtain the envelope (which represents the respiration) rather than the EMG waveform. Therefore, ECG removal was accomplished by detecting and deleting the QRS sections from the EMGdi signal. Once the QRS interference was removed, the signal was rectified and the envelope was obtained using a Butterworth low-pass filter of order 6 with a cutoff frequency of $1.5 \mathrm{~Hz}$. Breaths were detected from the reference effort belt and the respiration derived from EMGdi signal, in the same time window of 100 seconds. Various methods, including zero crossing and counting procedures, were experimentally tested in order to select the breaths detector. The method that showed the best results to detect the breathing oscillations was the advanced counting technique proposed by [11], which was also reported by the authors as the one with better performance. The idea of the algorithm is to calculate the amplitude of the breathing oscillations from the respiratory signal and consider only as a breathing the ones that exceed an amplitude threshold determine by the third quartile $\left(\mathrm{Q}_{3}\right)$ of the total amplitude values. Thus, if the oscillation amplitude is more than the $0.3 \times \mathrm{Q}_{3}$ (when analyzing a respiration signal) or $0.1 x \mathrm{Q}_{3}$ (in the case of HRV data), it is considered as a breathing [11]. After the respirations were detected, visual validation was done to identify and correct the misdetected breaths and determine the reference respiratory rate.

\section{RESULTS}

ECG signal obtained from the electrodes in the diaphragm is shown in Figure 2 (a). QRS complex is easily distinguished and the morphology of the wave can be identified. In Figure 2 (b), the raw EMGdi signal obtained is shown. However, due to the small amplitude of the muscle electric activity compared with the electrical impulses in the heart, the EMG signal is highly contaminated and cannot be distinguished. Nevertheless, after the removal of the QRS complex, electromyography activity of the diaphragm during respiration was acquired (Figure 2 (c)) and through rectification and low pass filtering, the breathing signal derived from EMGdi was obtained (Figure 2 (d)). There is a delay between this signal and the reference (Figure 2 (e)) generated by the conditioning of the EMGdi, but it can be observed an excellent correlation between both signals to show the breathing activity of the neonate.

Figure 3 represents the agreement between the RR intervals measured from the ECG lead obtained in the diaphragm compared with lead I (Figure 3(a)), lead II (Figure 3 (b)) and lead III (Figure 3(c)); using Bland-Altman plots. The resolution, with the sampling frequency of $1200 \mathrm{~Hz}$, was $0.83 \mathrm{~ms}$ explaining the empty spaces in the plots. Only one of the subjects showed RR intervals above $500 \mathrm{~ms}$ but showed excellent correlation to the reference ECG as is evident in Figure 3. Thus, the Bland-Altman plots showed the similarity between the RR intervals measurements in the diaphragm and the bipolar leads with no differences of more than four milliseconds. The mean difference between the RR intervals was $5.0875 \mathrm{e}-4 \mathrm{~ms}(S D=0.5111)$ with lead I, $0.0010 \mathrm{~ms}(S D=0.6453)$ with lead II and $1.8219 \mathrm{e}-13$ $(S D=0.6099)$ with lead III. The accuracy of the algorithm to detect the QRS complex was $99.39 \%$ for lead I, $90.09 \%$ for lead II, $99.66 \%$ for lead III and $100 \%$ for the ECG signals obtained from the diaphragm; mainly because of the fact that motion artifacts are reduced with the electrodes connected to the torso of the patient rather than in the limbs. 


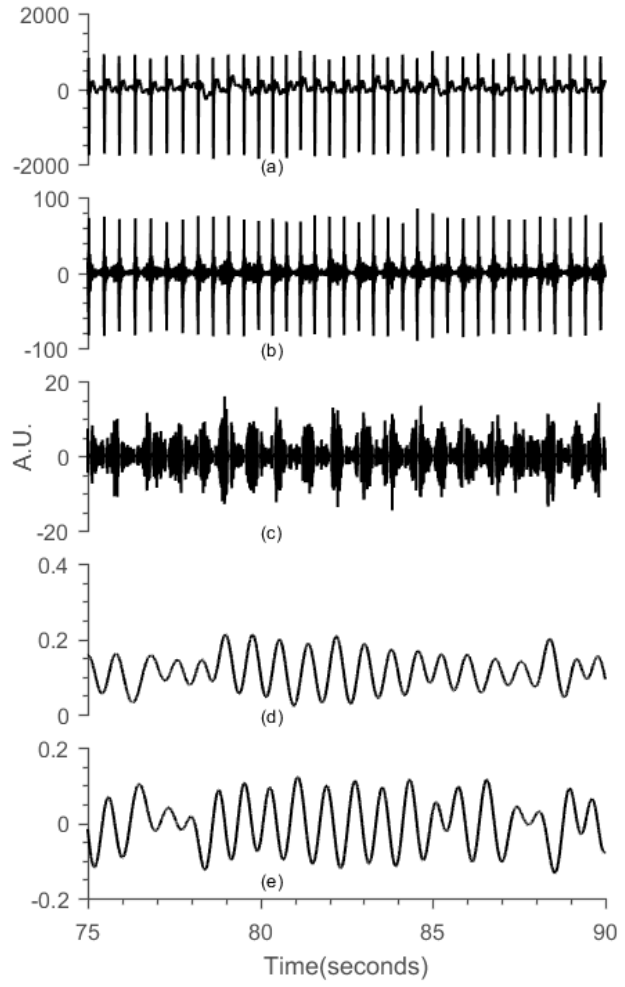

Figure 2. Signals obtained in one subject. (a) ECG signal between 0.5$40 \mathrm{~Hz}$ obtained from the diaphragm. (b) Raw EMGdi highly contaminated by ECG interference. (c) Filtered EMGdi signal with the removal of QRS complex. (d) Respiration derived from EMGdi signal. (e) Reference respiration signal from piezoelectric respiratory effort belt.

Table 1 shows the number of breaths detected in 100 seconds of data using two different thresholds with the advanced counting method proposed by [11], in the respiration signal derived from EMGdi and the reference from the piezoelectric respiratory effort belt. The first value in the parentheses indicates the false negatives (FN) identified by the algorithm and the second value the false positives (FP), compared with the breaths detected by visual inspection of the signals. As it can be noted, there were no FP using the EMGdi derived signal with a threshold of $0.3 \times Q_{3}$ and the number of FN is slightly less than in the belt signal. In Figure 2 it can be seen that the respiration waveform has oscillations of different amplitudes. Thus, these errors are decreased when the threshold in both signals is reduced, because lower amplitude oscillations are considered too. Nonetheless, the reduction in the threshold leads to an increase in the number of FP detected by the algorithm, especially in the respiration derived from EMGdi, where half of the FP were found in subjects 8 and 12 due to the presence of noise in the EMGdi breathing waveform. This FP could be reduced with the use of adaptive thresholds in the advance counting method.
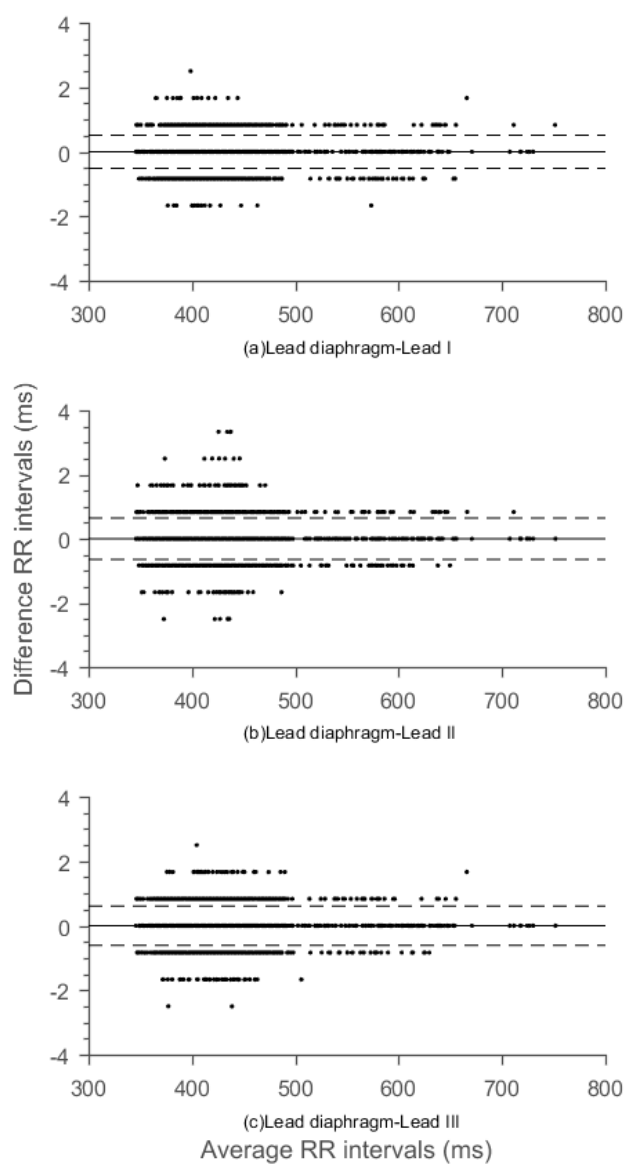

Figure 3. Bland-Altman plots of differences between RR intervals measurements in bipolar leads and ECG from the diaphragm, plotted against the average of the two measurements. Lines represent the mean and broken lines the standard deviations.

\section{DISCUSSION}

Signals obtained from the diaphragm using ECG can be employed to monitor heart rate without any significant changes or additional challenges. Traditional algorithms based on the well-known Pan-Tompkins detector can be used for R-peak detection in the ECG wave obtained from the diaphragm with excellent accuracy. On the other side, the respiration signals from EMGdi are highly contaminated with ECG interference and need to be filtered to obtain a reliable wave related with diaphragm contraction during breathing activity in neonates. Detection of breathings can be done in the EMGdi derived signal but better algorithms are needed to reduce the FN while maintaining the FP as low as possible. Selection of a proper threshold for breathing cycle detection depends on the requirement for specificity and sensitivity in the application. 
Table 1 Number of breaths detected using two different thresholds and the advanced counting proposed by [11]. See text for more explanation.

\begin{tabular}{ccccc}
\hline \multirow{3}{*}{ Subject } & \multicolumn{2}{c}{$\begin{array}{c}\text { No. of breaths } \\
\text { Th=0.3 } x \mathrm{Q}_{3}\end{array}$} & \multicolumn{2}{c}{$\begin{array}{c}\text { No. of breaths } \\
\text { Th=0.1 }\end{array} \mathrm{Q}_{3}$} \\
\cline { 2 - 5 } & $\begin{array}{c}\text { Respiration } \\
\text { from EMGdi }\end{array}$ & Belt signal & $\begin{array}{c}\text { Respiration } \\
\text { from EMGdi }\end{array}$ & Belt signal \\
\hline 1 & $104(16 / 0)$ & $98(22 / 0)$ & $111(9 / 0)$ & $112(10 / 2)$ \\
2 & $86(3 / 0)$ & $88(1 / 0)$ & $89(0 / 0)$ & $89(0 / 0)$ \\
3 & $96(10 / 0)$ & $105(3 / 2)$ & $104(3 / 1)$ & $102(4 / 2)$ \\
4 & $117(9 / 0)$ & $111(15 / 0)$ & $126(3 / 3)$ & $123(3 / 0)$ \\
5 & $73(0 / 0)$ & $73(0 / 0)$ & $74(0 / 1)$ & $74(0 / 1)$ \\
6 & $97(0 / 0)$ & $97(0 / 0)$ & $97(0 / 0)$ & $97(0 / 0)$ \\
7 & $77(4 / 0)$ & $75(6 / 0)$ & $82(1 / 2)$ & $80(2 / 1)$ \\
8 & $78(23 / 0)$ & $84(17 / 0)$ & $98(8 / 5)$ & $98(4 / 1)$ \\
9 & $88(5 / 0)$ & $91(2 / 0)$ & $95(0 / 2)$ & $93(0 / 0)$ \\
10 & $83(0 / 0)$ & $84(0 / 1)$ & $83(0 / 0)$ & $84(0 / 1)$ \\
11 & $66(1 / 0)$ & $66(1 / 0)$ & $66(1 / 0)$ & $67(0 / 0)$ \\
12 & $85(7 / 0)$ & $77(16 / 1)$ & $98(0 / 6)$ & $87(5 / 0)$ \\
13 & $82(5 / 0)$ & $82(5 / 0)$ & $87(1 / 1)$ & $87(1 / 1)$ \\
14 & $112(18 / 0)$ & $116(14 / 0)$ & $124(6 / 0)$ & $124(6 / 0)$ \\
Total & $1244(101 / 0)$ & $1247(102 / 4)$ & $1334(32 / 21)$ & $1317(35 / 7)$ \\
\hline
\end{tabular}

Combining ECG and EMGdi for cardiorespiratory monitoring allows to keep the connections with the newborn as low as possible while acquiring trustworthy signals. Injection of additional high-frequency current is no longer needed compared with the IPG technique that is currently used in most respiratory monitors and is not always reliable for doctors in the NICU, leading to manual observation and calculation of the respiratory rate. The method proposed here combine heart rate monitoring with the acquisition of a signal related directly with respiratory activity, and which can be used to detect episodes of apnea in neonates [7].Further studies should be done to analyze the signals obtained in cases of different breathing patterns and changes in heartbeats. A deeper analysis of the ECG waveform measured from the diaphragm is also needed to understand the changes generated by the movement of the electrodes from the traditional locations to the diaphragm.

\section{CONCLUSIONS}

Heart and respiration rate monitoring can be accomplished by acquiring the ECG and EMGdi simultaneously from the diaphragm in neonates without the need for extra sensors. Bland-Altman plots showed good agreement between the RR intervals measured from the diaphragm and the bipolar leads. Traditional algorithms for R-peak detection and heart rate computation can be applied on the ECG acquired from the diaphragm. Detection of breaths showed similar results in the EMGdi derived waveforms compared with the respiratory effort belt. The proposed method could be implement- ed in the NICU to monitor cardiorespiratory activity continuous and noninvasively.

\section{ACKNOWLEDGMENT}

We would like to express our gratitude to Hospital General de Medellín Luz Castro de Gutiérrez for the support in the data collection.

\section{CONFLICT OF INTEREST}

The authors declare that they have no conflict of interest.

\section{REFERENCES}

1. S. Shinichi, I.-N. Wako, I. Akira, K. Masanari, M. Shinobu, O. Kyoichi, I. Nobuya, T. Goro, and T. Tsutomu (2010) “Assessment of a new piezoelectric transducer sensor for noninvasive cardiorespiratory monitoring of newborn infants in the NICU," Neonatology, vol. 98, no. 2, pp. 179-190.

2. I. Smith, J. Mackay, N. Fahrid, and D. Krucheck (2011) "Respiratory rate measurement: a comparison of methods," Br. J. Health. Assist., vol. 5 , no. 1 , pp. $18-23$.

3. M. Folke, L. Cernerud, M. Ekström, and B. Hök (2003) "Critical review of non-invasive respiratory monitoring in medical care," Med. Biol. Eng. Comput., vol. 41, pp. 377-383.

4. C. Brüser, C. H. Antink, T. Wartzek, M. Walter, and S. Leonhardt (2015) "Ambient and unobtrusive cardiorespiratory monitoring techniques," IEEE Rev. Biomed. Eng., vol. 8, pp. 30-43.

5. J. M. Di Fiore, C. F. Poets, E. Gauda, R. J. Martin, and P. MacFarlane (2015) "Cardiorespiratory events in preterm infants: etiology and monitoring technologies," J. Perinatol., vol. 36, no. October, pp. 1-8.

6. E. J. W. Maarsingh, L. A. Van Eykern, A. B. Sprikkelman, M. O. Hoekstra, and W. M. C. Van Aalderen (2000) "Respiratory muscle activity measured with a noninvasive EMG technique : technical aspects and reproducibility," J. Appl. Physiol., vol. 88, pp. 1955-1961.

7. J. M. Ochoa, J. S. Osorio, R. Torres, and C. N. Mcleod (2009) "Development of an Apnea Detector for Neonates using Diaphragmatic Surface Electromyography," in 31st Annual International Conference of the IEEE EMBS, pp. 7095-7098.

8. J. Pan and W. J. Tompkins (1985) A Real-Time QRS Detection Algorithm, IEEE Trans. Biomed. Eng., vol. BME-32, no. 3, pp. 230236.

9. G. D. Clifford, "rpeakdetect." [Online]. Available: http://www.mit.edu/ gari/CODE/ECGtools/ecgBag/rpeakdetect.m. [Accessed: 10-Feb-2017].

10. Y. Deng, W. Wolf, R. Schnell, and U. Appel (2000) "New aspects to event-synchronous cancellation of ECG interference: An application of the method in diaphragmatic EMG signals," IEEE Trans. Biomed. Eng., vol. 47, no. 9, pp. 1177-1184.

11. A. Schäfer and K. W. Kratky (2008) "Estimation of breathing rate from respiratory sinus arrhythmia: Comparison of various methods," Ann. Biomed. Eng., vol. 36, no. 3, pp. 476-485.

Author: Marcela Tobón-Cardona

Institute: EIA University

Street: Calle 25 Sur 42-73

City: Envigado

Country: Colombia

Email: marcela.tobon@oulu.fi 